\title{
General Relativity in a $(2+1)$-Dimensional Space-Time: An Electrically Charged Solution
}

\author{
J. Richard Gott, III ${ }^{1}$, Jonathan Z. Simon ${ }^{1}$, and Mark Alpert ${ }^{1}$
}

Received August 15, 1985

\begin{abstract}
We have found a static electrically charged solution to the Einstein-Maxwell equations in a $(2+1)$-dimensional space-time. Studies of general relativity in lower dimensional space-times provide many new insights and a simplified arena for doing quantum mechanics. In $(2+1)$-dimensional space-time, solutions to the vacuum field equations are locally flat (point masses are conical sigularities), but when electromagnetic fields are present $T^{a b} \neq 0$ and the solutions are curved. For a static charge $Q$ we find $\vec{E}=Q \hat{r} / r$ and $d s^{2}=-\left(\kappa Q^{2} / 2 \pi\right) \ln \left(r_{c} / r\right) d t^{2}+\left(2 \pi / \kappa Q^{2}\right)\left[\ln \left(r_{c} / r\right)\right]^{-1} d r^{2}+r^{2} d \phi^{2}$ where $r_{c}$ is a constant. There is a horizon at $r=r_{c}$ like the inner horizon of the Reisner-Nordström solution. We have produced a Kruskal extension of this metric which shows two static regions (I and III) with $r<r_{c}$ and two dynamical regions (II and IV) with $r>r_{c}$. A spacelike slice across regions I and III shows a football-shaped universe with charge $Q$ at one end and $-Q$ at the other. Slices in the dynamical regions (II and IV) show a cylindrical universe that is expanding in region II and contracting in region IV. Electromagnetic solutions to the Einstein-Maxwell field equations in lower dimensional space-times can be used to provide new insights into Kaluza-Klein theories. In terms of the Kaluza-Klein theory, for example, electromagnetic radiation in a $(2+1)$-dimensional space-time is really gravitational radiation in the associated $(3+1)$ dimensional Kaluza-Klein space-time. According to Kaluza Klein theory the absence of gravitational radiation in $(2+1)$-dimensional space-time implies (correctly) the absence of electromagnetic radiation in $(1+1)$-dimensional space-time.
\end{abstract}

\section{INTRODUCTION}

In the first paper in this series, Gott and Alpert [1] examined general relativity in a $(2+1)$-dimensional space-time and explored point mass

${ }^{1}$ Department of Astrophysical Sciences, Princeton University, Princeton, New Jersey 08544. 
solutions and simple cosmological solutions. In the present paper, we will present an exact electrically charged solution to the combined Einstein-Maxwell equations. Studies of general relativity in a $(2+1)$ dimensional space-time carried out by a number of authors [1-5] have proven instructive in a number of ways. They provide examples of quasiregular singularities [1], serve as an arena in which simplified quantum mechanical calculations can be carried out [2-5] and illustrate some of the subtle ways in which the dimensionality of space-time affects physics. One interesting application was that the exact solutions for point masses in $(2+1)$-dimensional space-time $[1,2,3]$ led directly to exact solutions for strings in $(3+1)$-dimensional space-time Gott [6]. The first look at $(2+1)$-dimensional space-time was Abbott's [7] charming little book in 1884 called Flatland which described a whole universe with only twospatial dimensions. Recently Dewdney [8] explored how many aspects of everyday physics would behave in such a world. In what follows Latin indices $(a, b, m, n)$ run from 0 to 2 , the signature of the metric is $(-,+,+)$.

On the surface, general relativity retains many of its familiar features in a $(2+1)$-dimensional space-time. The metric $g_{m n}$, connection coefficients $\Gamma_{a b}^{m}$, and the Reimann curvature tensor $R_{a b c d}$ are defined in the usual way. Einstein's equation still holds $[1,2,3]: G_{m n}=\left(R_{m n}-\frac{1}{2} g_{m n} R\right)=\kappa T_{m n}$. In $(3+1)$ dimensions, the constant $\kappa$ is determined by the neccessity that Einstein's equations reduce to Newton's equations in the nonrelativistic limit, which forces $\kappa=8 \pi G$ ( $G$ is Newton's gravitational constant). In $(2+1)$ dimensions, as we shall see, there is no Newtonian limit, and so $\kappa$ remains an arbitrary constant. We note that in geometrized units, where $\kappa$ is dimensionless (and $c=1$ ), that $G_{u v}$ has units of curvature or (length) $)^{-2}$, and $T_{u v}$ has units of mass density or (mass) (length) ${ }^{-2}$, which makes mass dimensionless as well. The metric tensor $g_{m n}$ has six independent components, as does $R_{m n}$ and $R_{a b c d}$ (cf. Weinberg [9]). Since $R_{m n}$ and $R_{a b c d}$ have the same number of independent components, $R_{a b c d}$ can be given from $R_{m n}$ alone

$R_{a b c d}=g_{a c} R_{b d}-g_{a d} R_{b c}-g_{b c} R_{a d}+g_{b d} R_{a c}-\frac{1}{2}\left(g_{a c} g_{b d}-g_{a d} g_{b c}\right) g^{m n} R_{m n}$

This becomes especially important in the absence of mass, where $T_{m n}=0$. From Einstein's equation $R_{m n}=0$ also, and therefore $R_{a b c d}=0$ as well. This precludes any curvature at all in the vacuum, whether in the form of gravitational waves or attraction at a distance. (This is obviously different from the $(3+1)$-dimensional case, where $R_{\alpha \beta \gamma \delta}$ has 20 independent components while $R_{\mu \nu}$ has only 10 , and the Schwarzschild solution generates curvature outside the radius of a massive body.) The required flatness of space-time in the vacuum would seem to make the physics of gravity in $(2+1)$ dimensions almost trivial. 
It has been shown, however, by Gott and Alpert [1] and independently by Deser, Jackiw, and t'Hooft [2] and Giddings, Abbot and Kuchar [3] that, although the presence of mass cannot induce curvature per se at a distance, it does affect the space around the mass point. The space is locally flat, but conical in form. The metric around a point mass is $[1,2,3]$

$$
d s^{2}=-d t^{2}+\left(\frac{2 \pi}{2 \pi-\kappa M}\right)^{2} d r^{2}+r^{2} d \phi^{2}
$$

This metric is analogous to the Schwarzschild metric around a point mass in $(3+1)$ dimensions. This metric, however, does not lead to a black hole, and it can easily be seen that this metric corresponds to flat space-time by the simple transformations

$$
\begin{aligned}
r^{\prime} & =\left(\frac{2 \pi}{2 \pi-\kappa M}\right) r \\
\phi^{\prime} & =\left(\frac{2 \pi-\kappa M}{2 \pi}\right) \phi
\end{aligned}
$$

This gives the obviously flat metric

$$
d s^{2}=-d t^{2}+d r^{2}+r^{\prime 2} d \phi^{\prime 2}
$$

We must be careful to notice that the limits of $\phi^{\prime}$ are now different from the limits of $\phi: 0 \leqslant \phi \leqslant 2 \pi$, and $0 \leqslant \phi^{\prime} \leqslant 2 \pi-\kappa M$. The cone has an angle deficit of $\kappa M$ induced by the presence of mass. This has the effect of causing initially parallel light rays to converge as they pass on different sides of the particle, even though there is no curvature or Newtonian attraction. This type of singularity is called quasi-regular, having the property that as $r \rightarrow 0$, the Riemann curvature is bounded but the circumference is not equal to $2 \pi r$.

Another solution of the Einstein's equation in three dimensions is a static, dust-filled universe of radius $r_{0}[1,2]$. The metric is

$$
d s^{2}=-d t^{2}+r_{0}^{2}\left(d \theta^{2}+\sin ^{2} \theta d \phi^{2}\right)
$$

This is a universe with energy density $1 / \kappa r_{0}{ }^{2}$ but zero pressure (there is no attraction between the particles). The total area of the universe is $4 \pi r_{0}^{2}$, giving total mass $4 \pi / \kappa$, independent of the size of the universe (recall that mass is dimensionless). 
Electromagnetism also becomes simplified in $(2+1)$ dimensions.

$$
F^{m n}=\begin{array}{ccc}
0 & E_{1} & E_{2} \\
-E_{1} & 0 & B \\
-E_{2} & -B & 0
\end{array}
$$

Now the electric field $\vec{E}=\left(E_{1}, E_{2}\right)$ is still a vector, but the magnetic field $B$ is a scalar. It is easily seen that by extrapolating the metric into $(4+1)$ dimensions, the magnetic field would become a tensor with six independent components. We see that although the electric field $E$ can always be viewed as a vector, it is only in $(3+1)$ dimensions that the magnetic field happens to be a vector as well.

The equation of motion for a test charge of mass $m$ and charge $e$ is

$$
\frac{e}{m} F_{a}^{m} \frac{d x^{a}}{d \tau}=\frac{d^{2} x^{m}}{d \tau^{2}}+\Gamma_{a b}^{m} \frac{d x^{a}}{d \tau} \frac{d x^{b}}{d \tau}
$$

In $(3+1)$ dimensions Maxwell's equations are

$$
\begin{aligned}
F_{a \beta ; \gamma}+F_{\gamma a ; \beta}+F_{\beta \gamma ; a} & =0 \\
F_{; \beta}^{a \beta} & =k J^{a} \quad \text { where } \quad k=4 \pi
\end{aligned}
$$

The analogue of $(9)$ in $(2+1)$ dimensions would naturally be

$$
F_{a b ; c}+F_{c a ; b}+F_{b c ; a}=0
$$

For flat space-time the only nontrivial solution to this equation arises from the case that all indices are different, which gives

$$
0=\frac{-\partial E_{1}}{\partial y}+\frac{\partial E_{2}}{\partial x}+\frac{\partial B}{\partial t}=\operatorname{curl}(\vec{E})+\frac{\partial B}{\partial t}
$$

which is obviously analagous to the $(3+1)$-dimensional vector equation

$$
0=\nabla \times \vec{E}+\frac{\partial \vec{B}}{\partial t}
$$

except that with only two spatial dimensions the curl of a vector is a scalar, which gives only one equation, as opposed to the three equations implied by the vector equation (13). It should also be noted that $\nabla \cdot \vec{B}=0$, which is the other equation that arises from (9) in four dimensions, has no analogy in Flatland where $B$ is a scalar. From (10) we expect the analogous equation to be

$$
F_{; b}^{a b}=k J^{a}
$$


with the constant $k$ to be determined. For $a=0$, this reduces to Gauss' Law (again using Cartesian coordinates for flat space-time)

$$
\frac{\partial E_{1}}{\partial x}+\frac{\partial E_{2}}{\partial y}=\nabla \cdot \vec{E}=k \rho
$$

where $\rho$ is (surface) charge density. Just as Gauss' Law gives us an $r^{-2}$ dependency for the electric field of a stationary point charge in three spatial dimensions, it gives an $r^{-1}$ dependency in two spatial dimensions. Rewriting Gauss' Law in integral form

$$
\int \vec{E} \cdot \hat{n} d l=k Q
$$

and inserting $\vec{E}=Q \hat{r} / r$

$$
k Q=\int \vec{E} \cdot \hat{n} d l=\int_{0}^{2 \pi} \frac{Q}{r} r d \theta=2 \pi Q \quad \text { giving } \quad k=2 \pi
$$

Thus we can rewrite the $(2+1)$-dimensional counterpart of $(12)$ as

$$
F_{; b}^{a b}=2 \pi J^{a}
$$

Actually, the choice $2 \pi$ arises only from our decision to use Gaussian units. The factor of $k$ could always have been absorbed into the definition of the unit of charge, as is the case with mks units. It is also a common convention to set $k=1$, absorbing dimensional factors into the charge, but we will continue to use Gaussian units.

Setting $a=1,2$ in (18) we get (again with Cartesian coordinates)

$$
\begin{aligned}
& \frac{\partial B}{\partial y}-\frac{\partial E_{1}}{\partial t}=2 \pi J_{1} \\
& \frac{\partial B}{\partial x}-\frac{\partial E_{2}}{\partial t}=2 \pi J_{2}
\end{aligned}
$$

Which are analogous to Maxwell's equation in four dimensions

$$
\nabla \times \vec{B}-\frac{\partial \vec{E}}{\partial t}=2 \pi \vec{J}
$$

Apparently there is some sort of analogy to the curl of $B$, even when $B$ is a scalar. Equations 19 and 20 can even be expressed in a more compact 
equation if we introduce a new operator $R$, which acts on a vector by rotating it $90^{\circ}$ in the clockwise direction

$$
\begin{aligned}
R \vec{F} & =R\left(F_{1} \hat{x}+F_{2} \hat{y}\right)=F_{2} \hat{x}-F_{1} \hat{y} \\
R \nabla & =R\left(\frac{\partial}{\partial x} \hat{x}+\frac{\partial}{\partial y} \hat{y}\right)=\frac{\partial}{\partial y} \hat{x}-\frac{\partial}{\partial x} \hat{y} \\
\nabla \cdot(R \vec{F}) & =\left(\frac{\partial}{\partial x} \hat{x}+\frac{\partial}{\partial y} \hat{y}\right) \cdot\left(F_{2} \hat{x}-F_{1} \hat{y}\right)=\frac{\partial F_{2}}{\partial x}-\frac{\partial F_{1}}{\partial y}=\operatorname{curl}(\vec{F}) \\
R R \vec{F} & =R^{2} \vec{F}=-\vec{F} \\
(R \nabla) A & =R(\nabla A)
\end{aligned}
$$

This notation lets us express Maxwell's equations in Flatland as

$$
\begin{aligned}
\nabla \cdot \vec{E} & =2 \pi \rho \\
R \nabla B-\frac{\partial \vec{E}}{\partial t} & =2 \pi \vec{J} \\
\operatorname{curl}(\vec{E})+\frac{\partial B}{\partial t} & =0
\end{aligned}
$$

One other important set of equations involving the electromagnetic tensor is its relation to the stress-energy tensor. In four dimensions

$$
T^{\mu \nu}=\frac{1}{4 \pi}\left(g_{\alpha \beta} F^{\mu \alpha} F^{\nu \beta}-\frac{1}{4} g^{\mu \nu} F_{\alpha \beta} F^{\alpha \beta}\right)
$$

In three dimensions this becomes

$$
T^{m n}=\frac{1}{2 \pi}\left(g_{a b} F^{m a} F^{n b}-\frac{1}{4} g^{m n} F_{a b} F^{a b}\right)
$$

where again the change from $4 \pi$ to $2 \pi$ comes from the use of Gaussian units. That the factor of $\frac{1}{4}$ remains the same, regardless of the number of dimensions, is very important. For a proof of (31) see Alpert [10], or just follow a derivation of $(30)$ using the least-action principle from any textbook, but integrate over $d x^{2} d t$ instead of $d x^{3} d t$.

It is by these equations that all gravitational and electromagnetic interactions will be governed in $(2+1)$-dimensional space-time.

\section{AN EXACT STATIONARY CHARGED SOLUTION}

We now investigate the curvature induced in the space exterior to a static charge $Q$. When electromagnetic fields are present, space is no longer 
a vacuum-that is, $T^{a b} \neq 0$, so the space-time is curved. We consider the effects of a stationary, radial, electric field, $E_{r}=E(r), B=0, E_{\phi}=0$, which is static and axially symmetric (we use polar coordinates where the charge is centered at $r=0$ ). The only nonzero components of the electromagnetic field tensor are therefore $F^{01}=E(r), F^{10}=-E(r)$.

Maxwell's first equation for curved space-time

$$
F_{a b ; c}+F_{c a ; b}+F_{b c ; a}=0
$$

is satisfied trivially. Since we are considering the space outside a body of charge $Q$ we must find an expression for $E(r)$ that satisfies the source-free Maxwell equation

$$
F_{; b}^{a b}=0
$$

When $a=0$, we obtain

$$
0=F^{01}{ }_{, 1}+F^{01}\left(\Gamma_{01}^{0}+\Gamma_{11}^{1}+\Gamma_{12}^{2}\right)
$$

When $a=1$, we note that $F^{10}{ }_{, 0}=0$ (the solution is static) and obtain

$$
0=F^{10}\left(\Gamma_{10}^{1}+\Gamma_{00}^{0}+\Gamma_{02}^{2}\right)
$$

The $a=2$ equation is trivially satisfied.

Since we have limited ourselves to an axially symmetric, static electric field, the metric is time-independent and axially symmetric as well. Therefore we can adopt the Schwarzschild form

$$
d s^{2}=-A(r) d t^{2}+B(r) d r^{2}+r^{2} d \phi^{2}
$$

where $A$ and $B$ are functions of $r$ only. Since

$$
\left(\Gamma_{10}^{1}+\Gamma_{00}^{0}+\Gamma_{02}^{2}\right)=0
$$

the $a=1$ Maxwell equation is trivially satisfied. Substituting $E(r)$ for $F^{01}$ in the Maxwell $a=0$ equation yields

$$
\frac{d E(r)}{d r}+E(r)\left(A^{\prime} / 2 A+B^{\prime} / 2 B+1 / r\right)=0
$$

where ' indicates a derivative with respect to $r$. Dividing by $E(r)$ and integrating gives

$$
\ln E(r)=-\ln r-\left(\frac{1}{2}\right) \ln A-\left(\frac{1}{2}\right) \ln B+C^{\prime}
$$


(where $C^{\prime}$ is constant). Thus

$$
E(r)=\frac{C}{r A^{+1 / 2}(r) B^{+1 / 2}(r)}
$$

where $C$ is another constant. Substituting this result into (31) we find the only nonzero components of $T_{m}^{h}$ are

$$
T_{0}^{0}=T_{1}^{1}=-T_{2}^{2}=-\frac{C^{2}}{4 \pi r^{2}}
$$

Substituting these into Einstein's field equations yields $A(r)=-\left(\kappa Q^{2} / 2 \pi\right) \ln \left(r / r_{c}\right)=1 / B(r)$, and $E_{r}=Q / r$ where $r_{c}$ is a constant. The metric is thus

$$
d s^{2}=-\frac{\kappa Q^{2}}{2 \pi} \ln \left(\frac{r_{c}}{r}\right) d t^{2}+\frac{2 \pi}{\kappa Q^{2}}\left[\ln \left(\frac{r_{c}}{r}\right)\right]^{-1} d r^{2}+r^{2} d \phi
$$

The parameter $r_{c}$ determines the scale of the system analogous to $R=2 G M$ in the Schwarzschild metric. It is interesting to note, however, that $r_{c}$ is independent of $Q$, a dimensionless quantity. It is easily checked that $t$ is timelike and $r$ is spacelike only for $r<r_{c}$, but discussion of this is delayed until Section 3.

The only nonzero connection coefficients are

$$
\begin{aligned}
& \Gamma_{01}^{0}=\frac{1}{2 r}\left[\ln \left(\frac{r}{r_{c}}\right)\right]^{-1}=\Gamma_{10}^{0} \\
& \Gamma^{1}{ }_{00}=\left(\frac{\kappa Q^{2}}{2 \pi}\right)^{2} \frac{1}{2 r} \ln \left(\frac{r}{r_{c}}\right) \\
& \Gamma_{11}^{1}=\frac{-1}{2 r}\left[\ln \left(\frac{r}{r_{c}}\right)\right]^{-1} \\
& \Gamma^{1}{ }_{22}=\frac{\kappa Q^{2}}{2 \pi} r \ln \left(\frac{r}{r_{c}}\right) \\
& \Gamma_{12}^{2}=1 / r=\Gamma^{2}{ }_{21}
\end{aligned}
$$

The only nonzero component of $R_{b}^{a}$ is

$$
R_{2}^{2}=\frac{1}{r^{2}} \frac{\kappa Q^{2}}{2 \pi}
$$


and the Ricci scalar is

$$
R=R_{a}^{a}=R_{2}^{2}=\frac{\kappa Q^{2}}{2 \pi} \frac{1}{r^{2}}
$$

This result might seem curious at first because $R$, the Ricci curvature scalar, is always identically zero in four dimensions for electromagnetic fields. The vanishing of $R$ is not a general result, however, and in fact only occurs in four dimensions, as can be demonstrated

$$
\begin{aligned}
T & =T_{\alpha}^{\alpha}=\frac{1}{4 \pi}\left(g^{\alpha \gamma} g_{x \mu} F_{y \delta} F^{\mu \delta}-\frac{1}{4} \delta_{\alpha}^{\alpha} F_{y \delta} F^{\gamma \delta}\right) \\
& =\frac{1}{4 \pi}\left(F_{y \delta} F^{\gamma \delta}-\frac{1}{4} 4 F_{\gamma \delta} F^{\gamma \delta}\right)=0
\end{aligned}
$$

since

$$
\begin{aligned}
\kappa T_{\alpha}^{\alpha} & =R_{\alpha}^{\alpha}-\frac{1}{2} \delta_{\alpha}^{\alpha} R \\
\kappa T & =-R \\
R & =-\kappa T=0
\end{aligned}
$$

In three dimensions, however, the trace of the Kronecker delta is not 4, but 3 , and

since

$$
\begin{aligned}
T_{a}^{a} & =\frac{1}{2 \pi}\left(F_{c d} F^{c d}-\frac{1}{4} \delta_{a}^{a} F_{c d} F^{c d}\right) \\
T & =\frac{1}{2 \pi}\left(F_{c d} F^{c d}-\frac{1}{4} 3 F_{c d} F^{c d}\right) \\
T & =\frac{1}{8 \pi} F_{c d} F^{c d}
\end{aligned}
$$

$$
\begin{aligned}
\kappa T_{a}^{a} & =R_{a}^{a}-\frac{1}{2} \delta_{a}^{a} R \\
\kappa T & =-\frac{1}{2} R \\
R & =-2 \kappa T=-\kappa \frac{1}{4 \pi} F_{c d} F^{c d}
\end{aligned}
$$

It is very important that the factor of $\frac{1}{4}$ in the equation for $T_{b}^{a}$ be independent of the number of dimensions, as derived from the action principle.

For $r_{c}>r>r_{m}$ we can construct an embedding diagram for the solution at $t=0$ in a euclidean space with $d s^{2}=d x^{2}+d y^{2}+d w^{2}$ and where $r^{2}=x^{2}+y^{2}$ (see Fig. 1). The constant $r_{m}$ is given by

$$
r_{m}=r_{c} \exp \left(-2 \pi / \kappa Q^{2}\right)
$$

at $r=r_{m}, g_{11}=1$. For $0<r<r_{m}$ the solution at $t=0$ can be shown in an embedding diagram in a Minkowski space with $d s^{2}=-d w^{2}+d x^{2}+d y^{2}$ 

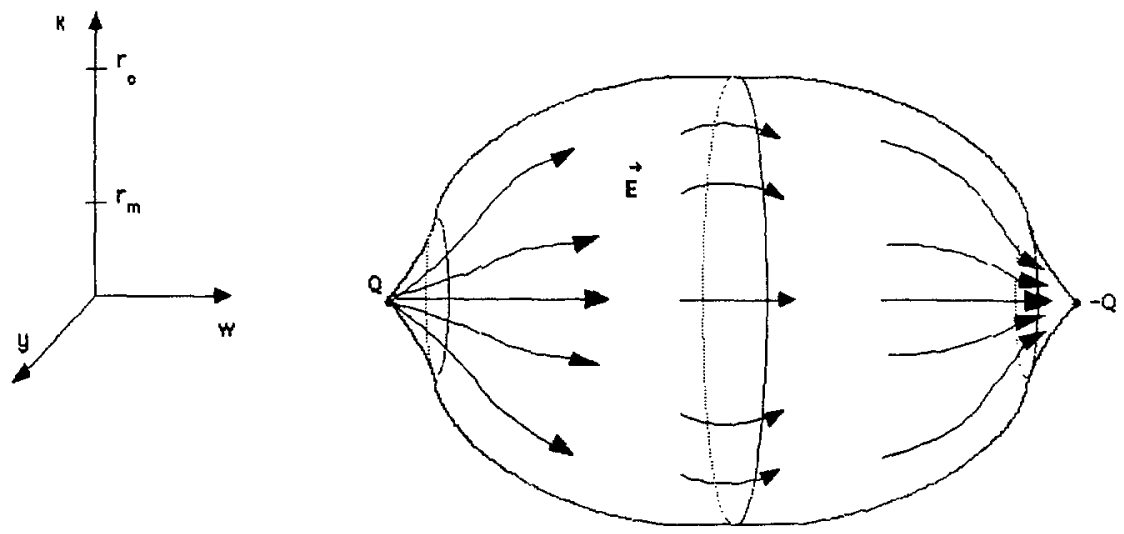

Fig. 1. Embedding diagram for the static solution (regions I, III). For $r>r_{m}$ the embedding space is euclidian $d s^{2}=d x^{2}+d y^{2}+d w^{2}$. For $r<r_{m}$ the embedding space is Minkowskian, $d s^{2}=d x^{2}+d y^{2}-d w^{2}$. We have put these pieces together to form a single diagram. The maximum radius is $r=r_{c}$.

where $r^{2}=x^{2}+y^{2}$; see Fig. 1. We may combine the two diagrams as shown in Fig. 1.

As will be shown in Section 3, this picture is incomplete.

\section{KRUSKAL EXTENSION}

Let us examine the behavior of the electrostatic metric in more detail. The metric is

$$
d s^{2}=-\frac{\kappa Q^{2}}{2 \pi} \ln \left(\frac{r_{c}}{r}\right) d t^{2}+\frac{2 \pi}{\kappa Q^{2}}\left[\ln \left(\frac{r_{c}}{r}\right)\right]^{-1} d r^{2}+r^{2} d \phi
$$

$$
\begin{array}{llll}
\text { as } & r \rightarrow 0 & g_{t t} \rightarrow-\infty & g_{r r} \rightarrow 0+ \\
\text { as } & r \rightarrow r_{c}- & g_{t t} \rightarrow 0- & g_{r r} \rightarrow \infty \\
\text { as } & r \rightarrow r_{c}+ & g_{t t} \rightarrow 0+ & g_{r r} \rightarrow-\infty \\
\text { as } \quad r \rightarrow \infty & g_{t t} \rightarrow \infty & g_{r r} \rightarrow 0-
\end{array}
$$

The behavior of the metric near $r=r_{c}, g_{00} \rightarrow 0$, and $g_{11} \rightarrow \infty$ is very similar to the behavior at the Schwarzschild radius of a black hole.

As $r$ becomes greater than $r_{c}, t$ changes from timelike to spacelike, and $r$ changes from spacelike to timelike. Qualitatively, it is the same as what happens at $r=2 G M$ in the Schwarzschild metric, except the change occurs 
going from small $r$ to large $r$ instead of the other way around. In fact, it is even more similar to the case of the Reisner Nordström black hole with charge $|Q|<M$, where there are an inner and outer horizon at $r_{-}$and $r_{+}$. If we could somehow force $r_{+} \rightarrow \infty$, then $r_{c}$ would be very much like $r_{-}$.

To be certain the black hole analogy holds we must be sure that the apparent singularity associated with the metric at $r=r_{c}$ arises purely from the coordinate system. First we check that the proper distance from $r=0$ to $r=r_{\mathrm{c}}$ is finite. If this distance is infinite, it hardly matters what happens at $r_{c}$ since no observer could arrive there in a finite time. To determine the proper distance, let us choose a radial curve from 0 to $r_{c}$ for which time is constant $(d t=d \phi=0)$.

$$
s\left(0, r_{c}\right)=\int_{0}^{r_{c}} d s=\int_{0}^{r_{c}}\left(\frac{2 \pi}{\kappa Q^{2}}\right)^{1 / 2}\left[-\ln \left(\frac{r}{r_{c}}\right)\right]^{-1 / 2} d r=\frac{\pi r_{c}}{Q}\left(\frac{2}{\kappa}\right)^{1 / 2}
$$

which is finite.

Second, we must be sure that the invariant curvature scalars of the metric are nonsingular at $r_{c}$. From Weinberg [9] we find that there are only three invariant curvature scalars associated with the metric in three dimensions

$$
R, R_{m n} R^{m n} \quad \text { and } \quad \frac{\operatorname{det} R}{\operatorname{det} g}
$$

Recalling that all $R_{m n}=0$ except $R_{22}=\kappa Q^{2} / 2 \pi$, the three invariant curvature scalars are

$$
\begin{gathered}
R=R_{2}^{2}=\kappa Q^{2} /\left(2 \pi r^{2}\right) \\
R_{m n} R^{m n}=R_{22} R^{22}=\left(\frac{\kappa Q^{2}}{2 \pi}\right)^{2} \frac{1}{r^{4}} \\
\frac{\operatorname{det} R}{\operatorname{det} g}=\frac{0}{-r^{2}}=0
\end{gathered}
$$

none of which shows any singularities at $r_{c}$, although there is a true singularity in the curvature at $r=0$.

Having now shown that the apparent singularity at $r_{c}$ is purely an artifact of the coordinate system $(t, r, \phi)$, we now find a new coordinate system, analogous to the Kruskal coordinates of a black hole, in which the apparent singularity does not appear (cf. Misner, Thorne, Wheeler [11]). 
For $r<r_{c}$ we can define Kruskal coordinates

$$
\begin{aligned}
& u=\left[-\ln \left(\frac{r}{r_{c}}\right) F\left(\frac{r}{r_{c}}\right)\right]^{1 / 2} \cosh \left(\frac{t \kappa Q^{2}}{4 \pi r_{c}}\right) \\
& v=\left[-\ln \left(\frac{r}{r_{c}}\right) F\left(\frac{r}{r_{c}}\right)\right]^{1 / 2} \sinh \left(\frac{t \kappa Q^{2}}{4 \pi r_{c}}\right)
\end{aligned}
$$

such that the metric becomes

$$
d s^{2}=\frac{8 \pi r_{c}^{2}}{\kappa Q^{2} F\left(r / r_{c}\right)}\left(-d v^{2}+d u^{2}\right)+r^{2} d \phi^{2}
$$

Where $r$ is now a function of $u$ and $v$, defined implicitly by the equation

$$
u^{2}-v^{2}=-F\left(r / r_{c}\right) \ln \left(r / r_{c}\right)
$$

and $F$ is the solution of the integral equation

$$
\int_{0}^{\rho} \frac{d x}{\ln x}=\ln |\ln (\rho) F(\rho)|
$$

The function $F(\rho)$ is defined

$$
F(\rho) \equiv \exp \left(\frac{\ln \rho}{1 \cdot 1 !}+\frac{(\ln \rho)^{2}}{2 \cdot 2 !}+\frac{(\ln \rho)^{3}}{3 \cdot 3 !}+\cdots\right)
$$

thus

$$
F^{\prime}(\rho)=F(\rho)\left[\frac{\rho-1}{\rho \ln \rho}\right]
$$

It is easily seen that any interesting behavior in $F(\rho)$ or $F^{\prime}(\rho)$ will be at $\rho=1$ or $\rho=0$. One can show that, in the limits as $\rho \rightarrow 1$ or $\rho \rightarrow 0, F(1)=1$, $F^{\prime}(1)=1, F(0)=0$, and $F^{\prime}(0)=0$.

Note that $F\left(r / r_{c}\right)$ is perfectly well-behaved at $r=r_{c}$ so that the metric is nonsingular as desired. The metric does become singular at $u^{2}-v^{2}=1$ $(r=0)$ where there is a real curvature singularity. Thus the metric is valid for all values of $(u, v)$ such that $u^{2}-v^{2}<1$. This region can be divided into quadrants:

$$
\begin{array}{rrrr}
\text { (I) } & u^{2}-v^{2}>0 & u>0 & \left(r<r_{c}\right) \\
\text { (II) } & u^{2}-v^{2}<0 & v>0 & \left(r>r_{c}\right) \\
\text { (III) } & u^{2}-v^{2}>0 & u<0 & \left(r<r_{c}\right) \\
\text { (IV) } & u^{2}-v^{2}<0, & v<0 & \left(r>r_{c}\right)
\end{array}
$$




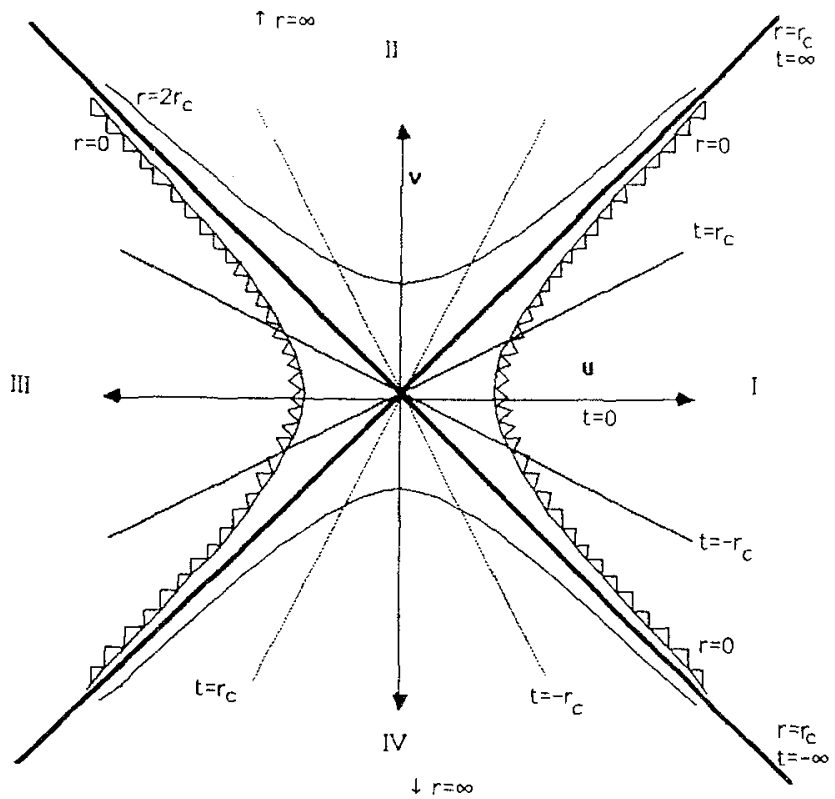

Fig. 2. Kruskal diagram of the electrically charged $(2+1)$-dimensional space-time. The singularities at $r=0$ containing the charges $+Q$ and $-Q$ are shown as saw-toothed curves. The embedding diagram of Fig. 1 is relevant for any $t=$ constant slice through regions $I$ and III. The embedding diagram of Fig. 3 is relevant for any $r=$ constant slice in region II or IV.

as shown in Fig. 2. The original $(r, t)$ coordinates for $r<r_{c}$ cover only quadrant I. We can define $(r, t)$ coordinates in each quadrant with $r$ always given by (71) and

$$
\begin{array}{ll}
t=\frac{4 \pi r_{c}}{\kappa Q^{2}} \tanh ^{-1}\left[\frac{v}{u}\right] & (\mathrm{I}, \mathrm{III}) \\
t=\frac{4 \pi r_{c}}{\kappa Q^{2}} \tanh ^{-1}\left[\frac{u}{v}\right] & (\mathrm{II}, \mathrm{IV})
\end{array}
$$

Where in each quadrant with these $(r, t)$ coordinates the metric is given by (62). Note that in (I, III), where $r<r_{c}, r$ is spacelike and $t$ is timelike, while in (II, IV), where $r>r_{c}, r$ is timelike and $t$ is spacelike.

One would like to show that the metric in terms of $u$ and $v$ is geodesically complete for timelike and null geodesics. While this will not be attempted here we can show that the metric is complete for a large family of timelike geodesics, namely ones for which $t=$ const and $\phi=$ const in quadrant II. We do this by showing that the total proper time between 
$v=0$ and $v=\infty$ for $u=0$ (which is the same as the total proper time between $r=r_{c}$ and $r=\infty$ for $t=0$ ) is infinite.

$$
\tau\left(r_{c, \infty}\right)=\int_{r_{c}}^{\infty} d \tau=\int_{r_{c}}^{\infty}\left(\frac{2 \pi}{\kappa Q^{2}}\right)^{1 / 2}\left[\ln \left(\frac{r}{r_{c}}\right)\right]^{-1 / 2} d r=\infty
$$

There are obvious similarities to the Schwarzschild case with the $u-v$ plane turned on its side. From this diagram we can see that the horizon at $r=r_{c}$ is not an event horizon but is qualitatively very similar to the inner horizon $r_{-}$of a charged black hole in four dimensions.

We can construct embedding diagrams of this space-time as follows. The first slice is along the hypersurface $t=0$ from one $r=0$ to $r=r_{c}$ and back to the other $r=0$ (i.e., from $u=-1$ to $u=+1$ along the line $v=0$ ). As the reader can see, this is just the same embedding diagram as in Fig. 1. Figure 3 is an embedding diagram for a spacelike slice in region II or IV. This is along one of the cylindrical hypersurfaces $r=$ const $>r_{c}$ which are shown as hyperbolas in Fig. 2. Along this hypersurface, the electric field lines are constant $(E=Q / r)$ and the field lines arise from charges that are infinitely far away (Gauss's law is still satisfied). Remember, however, that in this section of the universe $r$ is timelike; it is $t$ that is spacelike. As time progresses $r$ increases and the cylinder expands. Thus we can see that Fig. 3 quadrant II represents an evolving cosmology. This universe does not expand isotropically, since the Hubble constant is dependent on direction.

While this space-time is a solution of the classical Einstein-Maxwell equations, new problems will be brought in when quantum mechanical effects are added. One important effect would be particle-pair creation in the high electric fields encountered as $r \rightarrow 0$. If the solution is unstable then we could have a situation where only region IV forms similar to what happens in the Reisner Nordström solution in $(3+1)$-dimensional space-time.

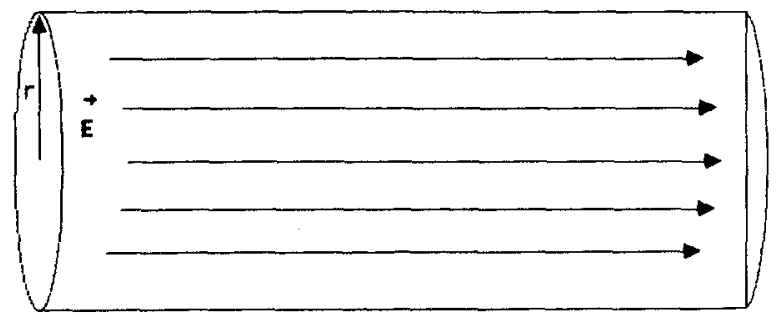

Fig. 3. The embedding diagram for an $r=$ constant slice in region II or IV is a cylinder. The embedding space is euclidean $d s^{2}=d x^{2}+d y^{2}+d w^{2}$. 


\section{APPLICATIONS TO KALUZA-KLEIN THEORIES}

One interesting application of such solutions to the Einstein-Maxwell equations in a $(2+1)$-dimensional space-time is in investigations of Kaluza-Klein theories. In its original version the Kaluza-Klein theory $[12,13]$ added one extra compact spacelike dimension to our $(3+1)$ dimensional space-time. The Einstein-Maxwell equations in $(3+1)$-dimensional space-time were then equivalent to just the Einstein equations in the $(4+1)$-dimensional Kaluza-Klein space-time. Thus the Einstein-Maxwell equations in a $(2+1)$-dimensional space-time $(x, y, t)$ must be equivalent to just the Einstein equations in the Kaluza-Klein $(3+1)$-dimensional space-time $(x, y, z, t)$. This is just the ordinary space-time of general relativity except that the $z$ dimension is compact and $g_{z z}=1$. It is easy to show that Maxwell's equations $(11,18)$ in a $(2+1)$-dimensional space-time admit solutions that are transverse electromagnetic waves with $\vec{E}$ perpendicular to the direction of motion and in phase with the scalar $B$ field. For example

$$
\begin{aligned}
E_{x} & =-E_{0} \cos [\omega(t-y)] \\
E_{y} & =0 \\
B & =E_{0} \cos [\omega(t-y)]
\end{aligned}
$$

is a solution representing an electromagnetic wave propagating in the $y$ direction at speed $c$. In the Kaluza-Klein $(3+1)$-dimensional formulation of this [14] we see that this solution is (in the limit of small $E_{0}$ [i.e., to first-order terms in $\left.E_{0}\right]$ ) produced by a gravity wave with $g_{\mu v}=\eta_{\mu \nu}+h_{\mu \nu}$ where $h_{t t v}=0$ except for

$$
h_{x z}=h_{z x}=(\kappa / \pi)^{1 / 2} E_{0} \omega^{-1} \sin [\omega(t-y)]
$$

This is a gravity wave propogating in the $y$ direction at speed $c$ with an " $x$ " polarization. The "+" polarization is not allowed due to the Kaluza-Klein constraint $g_{z z}=1$. Thus with the Kaluza-Klein unification of gravity and electromagnetism we see that the photons we see in a $(2+1)$ dimensional space-time are really just ordinary gravitons. Gravity waves in the Kaluza-Klein space-time propagating in the $x$ or $y$ directions look like electromagnetic waves and Kaluza's constraint that the metric coefficients be independent of $z$ means that any propogation must be perpendicular to the $z$ direction. Thus it is not surprising that we do not see any gravity waves or gravitons in the $(2+1)$-dimensional formulation of general relativity. The Kaluza-Klein gravitons are used to make photons and there are not any left to play the role of gravitons. This formulation can be 
extended to still lower dimensional space-times. The Einstein-Maxwell equations in a $(1+1)$-dimensional space-time are equivalent to just the Einstein equations in a $(2+1)$-dimensional space-time. Now we know that there are no gravitational waves and no gravitons in a $(2+1)$-dimensional space-time (the only solution of the vacuum field equations must be locally Minkowski space). Thus using the Kaluza-Klein formalism we expect this to imply that there are no electromagnetic waves and no photons in a $(1+1)$-dimensional space-time. Indeed this turns out to be the case. When we formulate Maxwell's equations in a $(1+1)$-dimensional space-time we find that the only nonzero components of the electromagnetic field tensor are $F^{01}=-F^{10}=E_{1}$. There is no $B$ field and no electromagnetic radiation. The $E$ field at a given location simply measures the number of charges to the left of you on the line minus the number of charges to the right of you. Shake a charge at a distance and it produces no change in the $E$ field at your location, unless and until the charge actually crosses your position. There is no action at a distance, no electromagnetic radiation, and no photons, just as the Kaluza-Klein theory would have predicted. More details of this have been presented in [14] and will be treated in an upcoming paper [15].

Given that we have obtained a solution to the Einstein-Maxwell equations in a $(2+1)$-dimensional space-time it would be interesting to look at the associated $(3+1)$-dimensional Kaluza-Klein solution. A study of geodesics in such a model representing charged and uncharged particles might provide some interesting insights.

Note. Shortly after completing work on the electrically charged solution we found that Deser and Mazur [16] had also obtained a solution. Their metric is static, is expressed in isotropic coordinates, and only applies in regions I and III. They did not find the Kruskal extension. They noted in passing that M. Melvin (private communication) had also obtained a solution in Schwarzschild coordinates, but gave no further details. Our static metric (eq. 42) which covers regions I and III is in Schwarzschild coordinates and was discovered by one of us (M. A.) in 1982 [10]. Our Kruskal metric (eq. 70) covers regions I, II, III, IV.

\section{NOTE ADDED IN PROOF}

Melvin's paper has now appeared in print [17]. We would also like to mention early work on point mass solutions by Staruszkiewicz [18]. His paper correctly noted that the point mass solution is conical, but not that the angle deficit is proportional to the mass. 


\section{REFERENCES}

1. Gott, J. R. and Alpert, M. (1984). Gen. Rel. Grav., 16, 243.

2. Deser, S., Jackiw, R., and t'Hooft, G. (1984). Ann. Phys., 152, 220.

3. Giddings, S., Abbot, J., and Kuchar, K. (1984). Gen. Rel. Grav., 16, 751.

4. Deser, S. (1984). Phys. Lett., 140B, 321.

5. Deser, S. and Jackiw, R. (1984). Ann. Phys., 153, 405.

6. Gott, J. R. (1985). Ap. J., 288, 422.

7. Abbott, E. A. (1884). Flatland, 6th ed. (Dover, New York) p. 1952.

8. Dewdney, A. (1980). Sci. Am., 243(1), 18-31.

9. Weinberg, S. (1972). Gravitation and Cosmology (John Wiley \& Sons, New York).

10. Alpert, M. (1982). Undergraduate thesis (Astrophysics), Princeton University.

11. Misner, C. W., Thorne, K. S., and Wheeler, J. A., 1973. Gravitation (W. H. Freeman, San Francisco).

12. Kaluza, T. (1921). Sitzungsberichte (Prevssische Akadamie Der Wissenschaften, Germany) p. 966.

13. Klein, O. (1926). Z. Phys., 37, 896.

14. Simon, J. Z. (1985). Undergraduate thesis (Physics), Princeton University.

15. Gott, J. R. and Simon, J. Z. (1985). In preparation.

16. Deser, S. and Mazur, P. O. 1985. Class. Quantum Grav., 2 , L51.

17. Melvin, M. A. (1986). Class. Quantum Grav., 3, 117.

18. Staruszkiewicz, A. (1963). Acta. Phys. Polin., 24, 734. 